\title{
KINEMATIC MODELING AND SIMULATION OF HUMAN ARM REACH
}

\author{
By \\ Mehrdad Zafari \\ Bachelor of Mechanical Engineering \\ Tabriz University \\ Tabriz, Iran \\ A project \\ presented to Ryerson University \\ in Partial fulfilment of the \\ requirement for the degree of \\ Master of Engineering \\ in the program of \\ Mechanical and Industrial Engineering.
}

Toronto, Ontario, Canada 2005

(C) Mehrdad Zafari 2001 


\section{UMI Number: EC52997}

\section{All rights reserved \\ INFORMATION TO USERS}

The quality of this reproduction is dependent upon the quality of the copy submitted. Broken or indistinct print, colored or poor quality illustrations and photographs, print bleed-through, substandard margins, and improper alignment can adversely affect reproduction.

In the unlikely event that the author did not send a complete manuscript and there are missing pages, these will be noted. Also, if unauthorized copyright material had to be removed, a note will indicate the deletion.

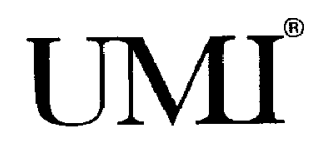

UMI Microform EC52997

Copyright 2008 by ProQuest LLC

All rights reserved. This microform edition is protected against unauthorized copying under Title 17, United States Code.

ProQuest LLC

789 East Eisenhower Parkway

P.O. Box 1346

Ann Arbor, MI 48106-1346 
I hereby declare that I am the sole author of this thesis or dissertation.

I authorize Ryerson University to lend this thesis or dissertation to other institutions or individuals for the purpose of scholarly research.

Signature 11.2 .902

I further authorize Ryerson University to reproduce this thesis or dissertation by photocopying or by other means, in total or in part, at the request of other institutions or individuals for the purpose of scholarly research.

Signature M. 2 apan 


\begin{abstract}
Modeling the human arm and calculation of the human arm reach are important for designing workspaces. An appropriate workspace design would reduce musculoskeletal disorders and wrist injuries in repetitive tasks. Among different human arm workspace designs and reach calculations, computer-based methods have recently attracted significant attention. This is because conventional experimental methods do not provide sufficient flexibility for interactive design modifications and integration of different aspects for workspace design. Despite advances in computer-aided design, many computer-based methods still rely on mock-ups and experimental set-ups. In this work, a computer-aided method is investigated for predicting the human arm reach. The kinematic modeling convention of Dennevit-Hertenberg is adopted for formulating the reach. Simulation results are provided to demonstrate the effectiveness of the method. The methodology enables visualization of the reach volume of the human arm and could be easily adjusted for different human operators.
\end{abstract}




\section{Acknowledgment}

I would like to express my gratitude to my supervisor Prof. Farrokh Janabi-Sharifi. This work and my higher education were not possible without his support. I would like to thank the thesis exam committee members for their valuable comments and discussions.

I would like to thank my wife who was patient with my long working hours and supported me during my MEng education. 


\section{TABLE OF CONTENTS:}

\section{Chapter 1. Introduction}

1.1 Motivation and Scope $\quad$....................................................

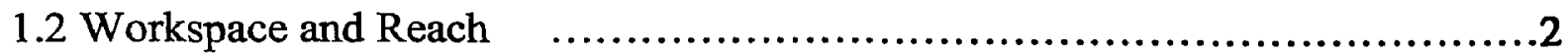

1.3 Previous Work $\quad$...........................................................

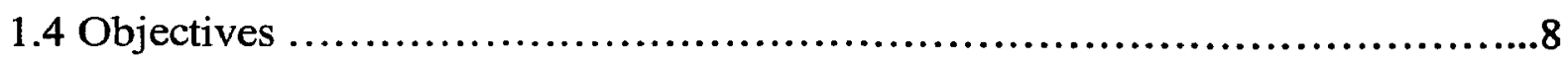

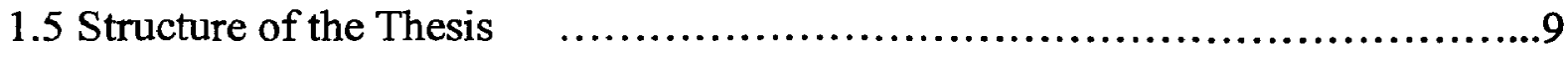

\section{Chapter 2. Robot Kinematics}

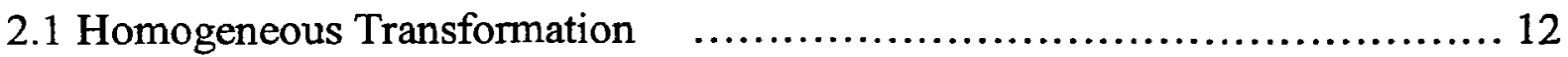

2.2 Forward Kineamtics $\quad$............................................. 13

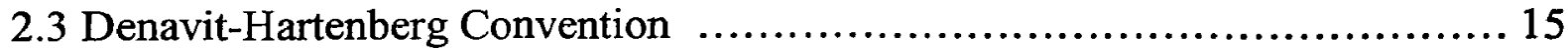

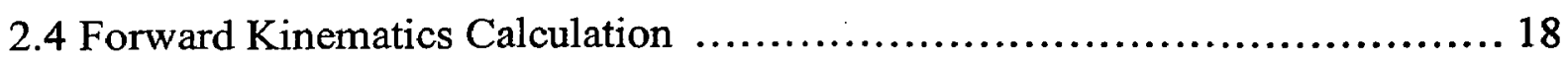

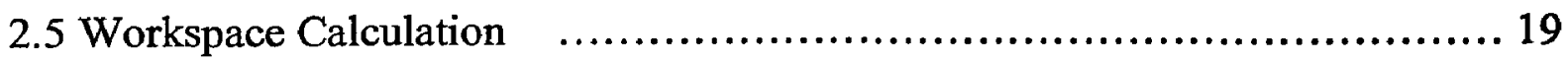

Chapter 3. Kinematic Modeling of Human Arm 21

3.1 Kinematic Structure $\quad$.............................................. 21

3.2 Kinematic Parameters $\quad$.............................................. 23

3.3 Forward Kinematics and Reach Envelope Algorithm $\quad$...................... 24

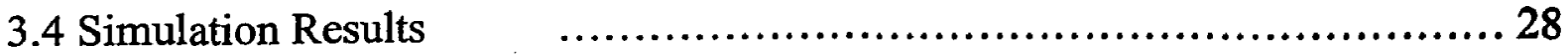


Chapter 4. Conclusions and Future Work

References 


\section{List of Figures}

1.1 Top view of the workspac

2.1 A kinematic representation of a 3 DOF planar robotic manipulator

2.2 Generalized link coordinate system with Denavit-Hartenberg kinematic convention 18

3.1 Kinematic modeling of human arm .23

3.2 Human arm envelope calculation algorithm .27

3.3 Reach envelope at $z=0$ plane of the base frame (all units in $\mathrm{cm}$ ) .30

3.4 Reach envelope at (a) $z=-15$, and (b) $z=-27$ of the base frame (all units in $\mathrm{cm}$ )

3.5 Reach envelope at (a) $z=-36$, and (b) $z=-45$ of the base frame (all units in $\mathrm{cm}$ ) 32

3.6 Reach envelope at $y=0$ plane of the base frame (all units in $\mathrm{cm}$ ) 33

3.7 Reach envelope at $y=-30$ plane of the base frame (all units in $\mathrm{cm}$ ) 


\section{List of Tables}

3.1 The Kinematic parameters for 4-DOF arm model .24 


\section{Chapter 1}

\section{Introduction}

\subsection{Scope and Significance}

Human workspace design plays an important role in reducing operation inefficiencie and work related health disorders. For instance, ignoring the ergonomic design of workspace can cause trauma disorders such as Repetitive Motion Injuries (RMIs), musculoskeletal disorders (MSDs), etc [1]. Among the common causes of work-related disorders and injuries are occurrences of joint angles away from neutral positions and poor posture with insufficient recovery time. For instance, in the computer industry, many assembly tasks are still manual, and wrist injuries (such as carpal tunnel syndrome of the wrist), shoulder tendonitis, lateral epicondylitis, tension neck syndrome are among the common disorders [2], [3].

In the recent decades there has been extensive work devoted to develop rules and principles pertaining to the design of workspaces [4], [5], [6]. Despite all the efforts and standards available, one source of difficulty is the poor presentation and integration of the developments into workspace design in an interactive and proactive manner. Consequently, computer-aided methods have been evolved to predict biomechanical strength, metabolic rate, and reach [7], [5]. However, many of the existing methods still require physical prototypes, 
and/or mock-ups. Ideally, the computer-aided methods must allow examination of the workspace before physical realization of the workspace or mock-up. Required features could then be incorporated into workspace designs to minimize risk of injuries and to maximize the work efficiency. Such computer-aided systems must accommodate modeling and analysis of the three-dimensional workspace, human anthropometries and postures. In particular, reach and visibility are amongst the most important features for the evaluation and feasibility of the workspace [8].

In this project, a computer-aided method is investigated for predicting the human arm reach. The kinematic modeling convention of Dennevit-Hertenberg [9] will be adopted for formulating the reach. The methodology will enable visualization of the reach volume of the human arm. The obtained model could be easily adjusted for different human operators.

\subsection{Workspace and Reach}

Workspace could be defined as "the total space in which an operator performs his/her duties" [10]. Many workspaces involve a fixed position for an operator, e.g., sitting behind a desk, or a panel. The workspace can be segmented into normal and maximal workspaces.

Normal workspace (Figure 1.1) could be defined as the "volume circumscribed by the horizontal forearm pivoting about a relaxed vertical upper arm" [10]. It is the space that can be reached by the forearm when the forearm pivots about a relaxed and fixed vertical upper arm. 
This is a low-energy consuming area and hence, materials, tools, and equipment are preferred to be located within this area.

Maximum workspace (Figure 1.1) is the "volume circumscribed from the minimum limb flexion to maximum limb extension of an operator" [10]. It is the maximum distance that can be reached by the fully extended arm as it pivots about the shoulder.

Reach of the human arm is the Cartesian space that can be reached by any combination of human arm joints.

In conventional methods, the front and practically important reachable workspace is considered (Figure 1.1). In more general terms, one might be interested in determining all possible reachable points. In major industrial designs, a workspace data sheet provides dimensions for establishing operator job relationships. For example, a classical approach of GM provides methods for obtaining average operators' sitting-standing principle and maximum horizontal reach at various elevations. The provided data sheet is used to determine shoulder pivot points on a planar view of the designer's drawing. A compass is then set to the horizontal displacement corresponding to the elevation being evaluated. The compass is then pivoted from the shoulder pivot points previously established on the drawing. The maximum workspace will be then the area enclosed within the radius. Such non-computer based approaches cannot be easily adopted for a new operator or workspace. Moreover, they are very simplistic, and conservative, and usually ignore good portions of the workspace. In the workspace calculation, the forearm and upper arm dimensions, as well as the joints limitations, must be considered. 
From a medical perspective, reach and workspace calculation to quantify the changes and limitation due to injuries or diseases is also important. For instance, treatment techniques for arm impairments would require monitoring of the arm wokrspace and reach [11]. In clinical studies, standard flexion and extension are used for evaluation of impairement after brain injury [12]. Despite the objective measures proposed, [13], [14], for a given constraint in a particular direction, the proposed measures cannot identify the entire range of the limitations. The clinical methods [15] are also limited by the number of Degrees of Freedom (DOFs) and the numerical methods used.

The focus of this work will be on human reach study that is based on the calculation of maximum workspace. The developed method can be easily adopted, though, for normal workspace calculation of both arms. The method allows ease of interfacing and visualisation.

The human reach calculation has many applications. For instance, it allows engineers to design the industrial workspace and to place tools and parts inside the workspace such that the risk of injuries could be minimized. Similarly, in cockpit and automobile interior design, an automatic reach calculation facilitates the placement of the instruments and dials in locations that are easy to reach. In general, complete reach modelling and envelope identification is important [16] for:

- Understanding neural strategies to allow posing the hand during voluntary reaching movements.

- Quantifying the functional potential of a joint.

- Facilitating the selection of ergonomic postures to reduce joint stresses. 


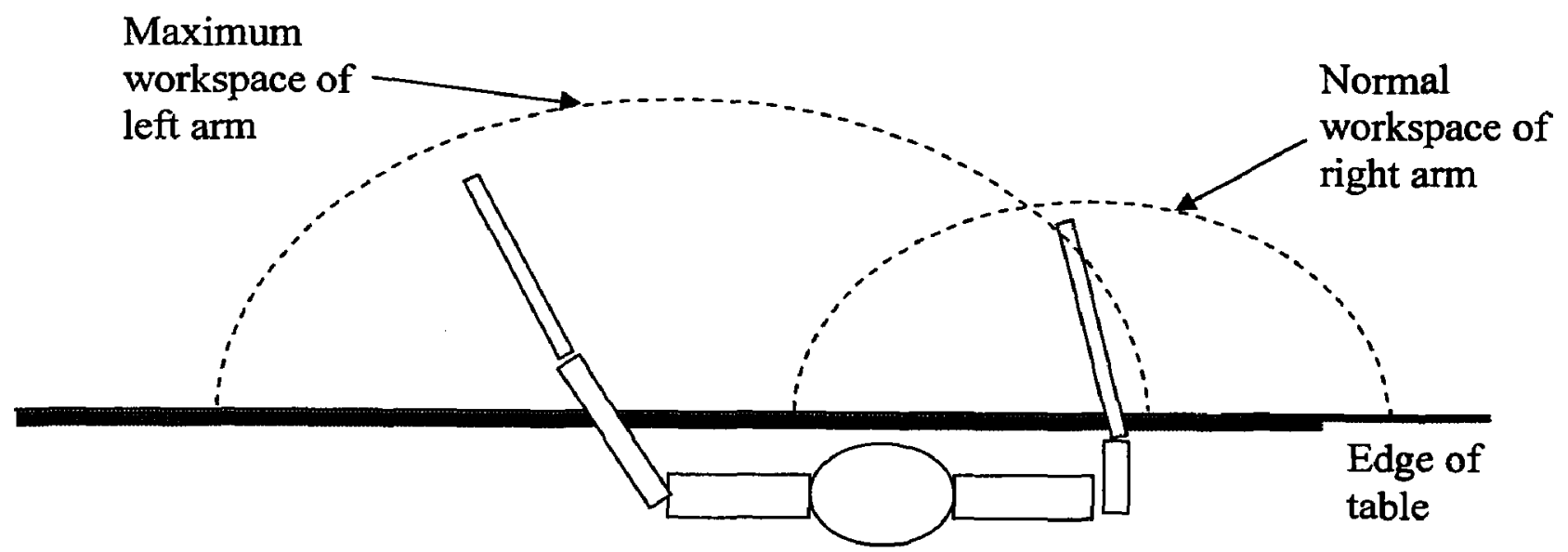

Figure 1.1 Top view of the workspace.

\subsection{Previous Work}

Conventional approaches used to rely on experiments within real workspaces or mockups. Some of the recent methods still rely on traditional techniques. With conventional methods, human reach can be measured by observing if the human hand simply reaches or fails 
to reach a specific point in the Cartesian (physical) space. Statistical and empirical mathematical models can then be derived to approximate the reach space boundaries. Examples of such approaches could be found in [10], [17], [18]. In [10], Farley determined the normal horizontal workspace for the right hand. In his experiment, only the forearm was extended during movement and the upper arm remained at the side of the body in a natural and vertical position until it started to swing away as the hand moved toward the outer part of the working area. The work of Farley was extended by Squires [19] who did not assume a fixed elbow position and allowed a circular path for the elbow as the forearm pivoted. The horizontal position of the reach was approximated by two sinusoidal functions. Later, it was concluded that the simple shapes of reach obtained do not confirm to various percentiles. In particular, initial and terminating angles that were assumed for the angle between the forearm and the table edges (i.e., 42 and 65 degrees, respectively) (Figure 1.1) did not correspond to many individuals [20], [18]. Later, the work of Squires was refined in [20] and [18] to give a more accurate normal horizontal workspace and parametric equations were developed to approximate the reach. In particular, it was found that relevant anthropometric parameters varied from individual to individual and percentile values could not be used in parametric equations. Squirel's equation was found to overestimate to the right and underestimate to the left of the body median, when investigated experimentally. In summary, conventional methods are difficult to implement, are usually mock-up based, provide only rough approximation of the reach and workspace, involve only limited degrees of freedom of the arm, and cannot be easily adopted for computer simulation or visualization of a virtual workspace. To overcome these deficiencies, computer-aided methods have been proposed. 
New methods are based on computer models for ease of visualization and simulation. Examples of available computer-aided systems are: SAMMIE [7], and Deneb/ERGO [21]. Many of these systems use different terminology, special structures and modeling techniques than commercially available CAD systems. Moreover, many of the systems are not accessible to many designers because they have been developed for special applications (military aircraft or automobiles), expensive, and cannot run on PCs [5]. Some of the computer-based methods assume only limited DOFs. Although the main concern in many manual tasks has been repetitive strain injuries of the hand and the wrist, the whole arm including shoulder girdle and glenohumeral joint are subject to injuries [31]. In addition to wrist injuries (such as carpal tunnel syndrome of the wrist), shoulder tendonitis, lateral epicondylitis, tension neck syndrome are also common [2], [3]. Therefore, the whole arm is required to be modeled.

Another concern has also been the fact that many of the previous approaches have used average population data for modeling and do not allow for differences between people [22]. Hence, the developed model must facilitate incorporation of individual arm data.

Based on the literature survey, it can be concluded that a good workspace calculation method must meet the following requirements:

- Should be readily adaptable to many tasks and workspace designs.

- Must integrate individual data rather than average population data.

- Must consider major degrees of freedom of the arm.

- Must not require expensive tools or computing systems, and could run on regular PCs. 
- Finally, the user must be able to enter required data and determine the workspace readily.

Recently, in order to address some of the above issues, there has been a trend towards the use of robot kinematics for modeling the human arm and calculation of the human arm reach. A good discussion on reachable space determination using inverse kinematics is given in [23]. Examples of recent works include [24], and [25]. The work presented in this project relies on robot kinematic modeling of the human arm. However, it is different than the analytical work presented in [24] that is limited to the structures with a few DOFs. The work in [25] formulates the problem of reach as the rank-deficiency determination of the arm Jacobian matrix. Their approach, however, requires complicated sweeping methods and non-automated inspection of the workspace interior for removal of redundant planes.

\subsection{Objective}

The objective of this work is to use robot kinematics to model the human arm and to calculate the human arm workspace using forward kinematics. The method will be formulated in such a way that it could

- Readily be adaptable to many tasks and workspace designs.

- Integrate individual operator's data rather than average population data.

- Encompass major degrees of freedom of the arm. 
- Be computationally simple and would not require expensive tools or computing systems, and could run on regular PCs.

- Provide easy interface for the user in order to enter required data and determine the workspace.

This will be the main contribution of this work.

\subsection{Structure of the Report}

In Chapter 2, the fundamentals of robot kinematics and Denavit-Hartenberg notion will be reviewed. The arm model will be developed in Chapter 3. Also, the simulation results will be given to show the human reach envelope. Discussion and conclusions will be presented in Chapter 4. 


\section{Chapter 2}

\section{Robot Kinematics}

A robotic manipulator usually consists of a series of links connected by joints. Robotic joints can be represented by a combination of revolute and prismatic types [26]. The structured arrangement of links and joints forms a kinematic chain (Figure 2.1). The very first link is connected to the base (link 0) and the last link is attached to the end-effector (gripper or tool). There is a base coordinate frame that is usually used as the robot world coordinate frame. Each joint is formulated to have a single degree of mobility (an angle) that constitutes a joint variable. Joint variables form the robot joint vector $q$, and the end-effector is located by its position and orientation with respect to the world coordinate frame, denoted by vector $\boldsymbol{x}$. For example, in Figure 2.1,

$$
x=\left[\begin{array}{l}
x \\
y \\
\theta
\end{array}\right] \text {, and } q=\left[\begin{array}{l}
\mathrm{q}_{1} \\
\mathrm{q}_{2} \\
\mathrm{q}_{3}
\end{array}\right] \text {. }
$$




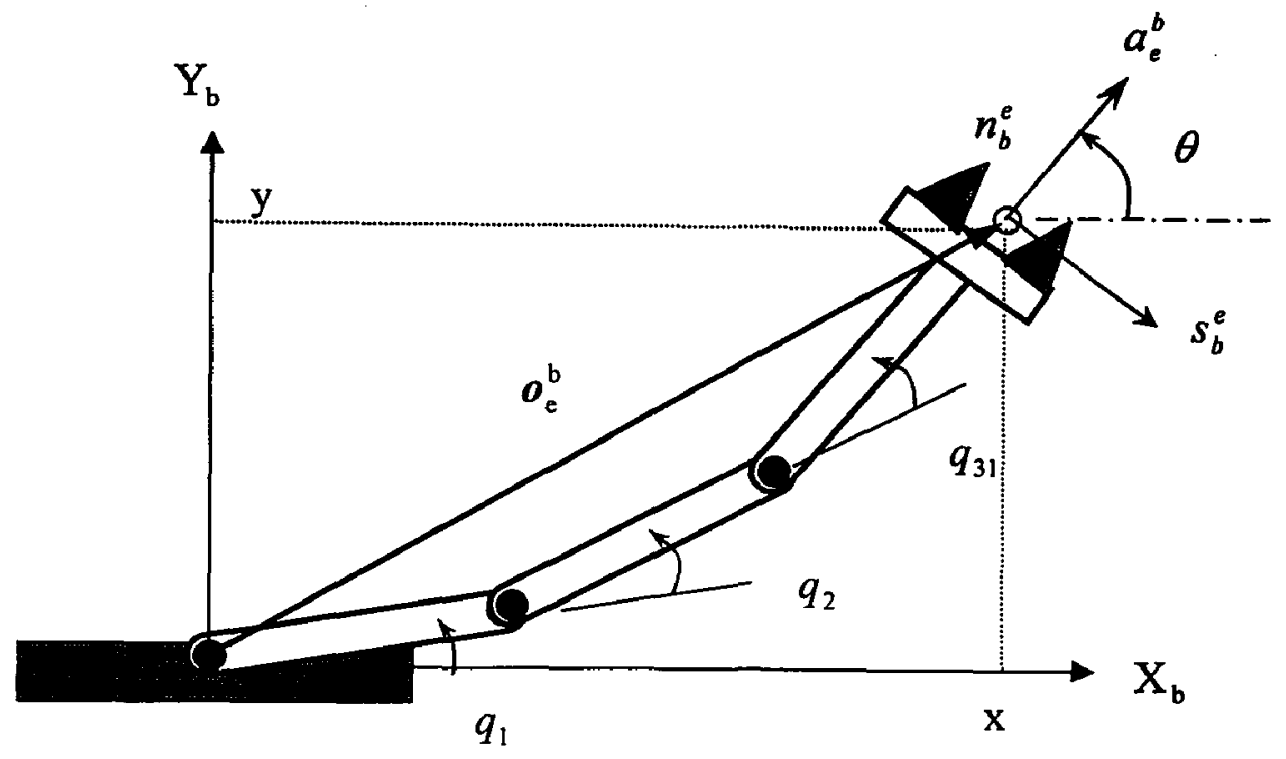

Figure 2.1 A kinematic representation of a 3 DOF planar robotic manipulator.

Robot kinematics is the study of the motion of a robot with respect to a certain coordinate system without any consideration for moments and forces. In robotics, robot kinematics is used to relate the robot joint variables vector $\boldsymbol{q}$ to the robot end-point pose (position and orientation) vector $\boldsymbol{x}$. Two robot kinematics issues include robot forward and inverse kinematic problems. In robot forward kinematics, the problem is to find the end-point pose vector $\boldsymbol{x}$ for a given robot joint variables vector q. In inverse kinematics, the problem is to find the possible robot joint variables vector $q$ for a given end-point pose vector $x$. In this report, the focus will be on the forward kinematics calculation. For this purpose, the homogeneous transformation concept will prove useful. 


\subsection{Homogeneous Transformation}

The position of a rigid body can be expressed by defining the position of a fixed point on the body with respect to a reference frame, and its orientation can be described in terms of the components of the unit vectors of a frame attached (at the same point) to the body, with respect to the same reference frame. For instance, in Figure 2.1, the position of the end-point can be expressed by $[x, y]^{T}$ and its orientation can be expressed by defining the unit vectors $\left[n_{\mathrm{e}}^{\mathrm{b}}, s_{\mathrm{e}}^{\mathrm{b}}, a_{\mathrm{e}}^{\mathrm{b}}\right]$. A homogeneous transformation is defined as a $4 \times 4$ matrix that generally represents translation, rotation, and scaling of rigid bodies in space. Assume two frames, 1 and 2, attached to two links. The homogeneous transformation matrix can be formed by defining the position vector of the origin of one body (2) with respect to the other body (1), i.e., $\boldsymbol{o}_{2}^{1}$, and the rotation matrix expressing the unit vectors of frame 2 with respect to frame 1, i.e., $\boldsymbol{R}_{2}^{1}$. Therefore, a homogeneous matrix relating these two consecutive frames $\{i-1\}$ and $\{i\}$ will be [26]:

$$
\boldsymbol{T}_{i}^{i-1}=\left[\begin{array}{cc}
\boldsymbol{R}_{i}^{i-1} & \boldsymbol{O}_{i}^{i-1} \\
\mathbf{0}^{\mathrm{T}} & \mathbf{1}
\end{array}\right]
$$

In general, if one considers normal, slide, and approach coordinate axes attached to the end-point (Figure 2.1), the rotation matrix will be: 


$$
R_{e}^{b}=\left[\begin{array}{lll}
\left(n_{\mathrm{e}}^{\mathrm{b}}\right)_{\mathrm{x}} & \left(s_{\mathrm{e}}^{\mathrm{b}}\right)_{\mathrm{x}} & \left(a_{\mathrm{e}}^{b}\right)_{\mathrm{x}} \\
\left(n_{\mathrm{e}}^{\mathrm{b}}\right)_{\mathrm{y}} & \left(s_{\mathrm{e}}^{\mathrm{b}}\right)_{\mathrm{y}} & \left(a_{\mathrm{e}}^{b}\right)_{\mathrm{y}} \\
\left(n_{\mathrm{e}}^{\mathrm{b}}\right)_{\mathrm{z}} & \left(s_{\mathrm{e}}^{\mathrm{b}}\right)_{\mathrm{z}} & \left(a_{\mathrm{e}}^{b}\right)_{\mathrm{z}}
\end{array}\right]
$$

Obviously, the homogeneous transformation matrix will be a function of joint variables. For example, in Figure 2.1, if one considers the frame attached to the end-point and the base frame, the homogeneous transformation matrix will be:

$$
\boldsymbol{T}_{e}^{b}=\left[\begin{array}{cccc}
0 & \sin \theta & \cos \theta & x \\
0 & -\cos \theta & \sin \theta & y \\
1 & 0 & 0 & 0 \\
0 & 0 & 0 & 1
\end{array}\right]
$$

\subsection{Forward Kinematics}

The problem of forward kinematics is to find position and orientation of the end-effector $\boldsymbol{x}$ for a given joint variables $q$, i.e., a nonlinear mapping from ( $n$-dimensional) joint space to $(m-$ dimensional) operation (Cartesian) space, summarized by

$$
\boldsymbol{x}=\boldsymbol{k}(q),
$$

where the vector function $k($.) represents forward kinematics. It should be noted that the representation of orientation in $x$ can be either done through homogeneous transformation (i.e., 
the rotation matrix $\boldsymbol{R}$ ) or explicitly by Euler angles describing the rotation of the end-effector frame with respect to the base frame. In the latter case, it will be:

$$
\boldsymbol{x}=\left[\begin{array}{l}
0 \\
\phi
\end{array}\right],
$$

where $\boldsymbol{o}$ is the position vector and $\phi$ denotes the orientation of the end-effector expressed explicitly by Euler angles. Computation of $\phi(q)$ is not straightforward and requires the computation of the rotation matrix. Therefore, the homogeneous transformation representation of rotation is more common and will be adopted in this work.

A serial manipulator with $n+1$ links connected by $n$ joints is considered. Link 0 is fixed to the stationary base. It is also assumed that each joint has a single degree of mobility. An open kinematic chain is formed where each joint connects two consecutive links. Therefore, the forward kinematic solution is obtained by considering the kinematic description between consecutive links and then deriving overall the kinematic solution in a recursive fashion. Therefore, starting from the base frame $\{b\}$ and continuing to the end-effector, the coordinate transformation describing the pose of the frame $\{e\}$ with respect to frame $\{b\}$ will be given by:

$$
T_{e}^{b}(q)=T_{1}^{0}\left(q_{1}\right) T_{2}^{1}\left(q_{2}\right) T_{3}^{2}\left(q_{3}\right) \ldots T_{n}^{n-1}\left(q_{n}\right) T_{e}^{n}
$$

Therefore, the computation of the forward kinematics function is recursive and is obtained in a systematic manner by simple products of the homogeneous transformation matrices $T_{i}^{i-1}\left(q_{i}\right)$ for $(i=1,2, \ldots, n)$. Note that the last homogeneous transformation matrix is constant 
describing the pose of the end-point frame $\{e\}$ with respect to frame $\{n\}$. The only issue remaining is a systematic and efficient way of calculating each homogeneous transformation matrix. The Denavit-Hartenberg convention provides a feasible solution to the problem.

\subsection{Denavit-Hartenberg Convention}

Based on the previous section discussion, one can conclude that the problem of the forward kinematics calculation can be reduced to determining two frames attached to two consecutive links and to calculate the coordinate transformation between these two frames. In order to calculate rotation and translation between two frames of adjacent links, several conventions have been introduced. Those conventions include the Denavit-Hartenberg (D-H) convention, the Sheth-Uiker (S-U) notation, and Spherical-Euler transformation (SET) [9], [27], [28]. Among these conventions, the Denavit-Hartenberg [9] convention is more popular since it provides a systematic method of frame attachment and coordinate transformation between two frames using homogeneous transformation. In fact, the $\mathrm{D}-\mathrm{H}$ convention uses the minimum number of parameters to describe the kinematic relationship between two coordinate frames.

Taking a general schematic diagram of two links (Figure 2.2), axis $i$ would denote the axis of the joint connecting link $i-1$ to link $i$. Assume that the coordinate frame $\{i-1\}$ has already been assigned. Then the frame $\{i\}$ can be assigned as follows:

- Select axis $z_{i}$ along the axis of motion of joint $i+1$. 
- Locate the origin of frame $\{i\}$, i.e. $O_{i}$ at the intersection of axis $z_{i}$ with the common normal to axes $z_{i-1}$ and $z_{i}$. Similarly, point $O_{i}^{\prime}$ is located at the intersection of the common normal with axis $z_{i-1}$.

- Choose axis $x_{i}$ along the common normal to axes $z_{i-1}$ and $z_{i}$ with direction away from joint $i$ to joint $i+1$.

- Choose axis $y_{i}$ to form a right-hand frame.

Note that for frame $\{0\}$, only the direction of axis $z_{0}$ is specified and $O_{0}$ and $x_{0}$ can be selected arbitrarily. For frame $\{n\}, x_{n}$ is normal to axis $z_{n-1}$ but since there is no joint $n+1, z_{n}$ is not uniquely defined. Since the last joint is usually revolute, $z_{n}$ is aligned with the direction of $z_{n-1}$. Also, when two consecutive axes become parallel, the common normal between them will not be uniquely defined. Another exception is when two consecutive axes intersect. In this case, the direction of $x_{i}$ will be arbitrary. The above minimal indeterminacy could be exploited to simplify the frame assignment in many practical robotic cases.

After the frame assignment, the D-H kinematic parameters between frames $\{i\}$ and $\{i-1\}$ (Figure 2.2) are determined as follows:

- $a_{i}$ : link length or distance between $O_{i}$ and $O_{i}^{\prime}$.

- $d_{i}$ : joint distance or coordinate of $O_{i}^{\prime}$ along $z_{i-1}$.

- $\alpha_{i}$ : link twist or angle between axes $z_{i-1}$ and $z_{i}$ about axis $x_{i}$, positive in counterclockwise rotation. 
- $\theta_{i}$ : joint angle or the angle between axes $x_{i-1}$ and $x_{i}$, positive in counter-clockwise rotation.

Out of the four parameters, two of them $\left(a_{i}\right.$ and $\left.\alpha_{i}\right)$ are always constant and depend on the geometry of link $i$. Out of the two remaining parameters, one is also constant for each joint type, i.e., for a revolute joint, $d_{i}$ is constant, while for a prismatic joint, $\theta_{i}$ is constant. Once the coordinate frames $\{i-1\}$ and $\{i\}$ have been assigned, it can be proven [26] that the homogeneous transformation matrix relating frames $\{i-1\}$ and $\{i\}$ will be:

$$
\boldsymbol{T}_{i}^{i-1}\left(q_{i}\right)=\boldsymbol{T}_{i^{*}}^{i-1} \boldsymbol{T}_{i}^{i^{*}}=\left[\begin{array}{cccc}
\cos \theta_{i} & -\sin \theta_{i} \cos \alpha_{i} & \sin \theta_{i} \sin \alpha_{i} & a_{i} \cos \theta_{i} \\
\sin \theta_{i} & \cos \theta_{i} \cos \alpha_{i} & -\cos \theta_{i} \sin \alpha_{i} & a_{i} \sin \theta_{i} \\
0 & \sin \alpha_{i} & \cos \alpha_{i} & d_{i} \\
0 & 0 & 0 & 1
\end{array}\right]
$$

The above equation is for the transformation from frame $\{i\}$ to frame $\{i-1\}$ and is only a function of joint variable $q_{i}$ that will be either $\theta_{i}$ (for a revolute joint) or $d_{i}$ (for a prismatic joint). 


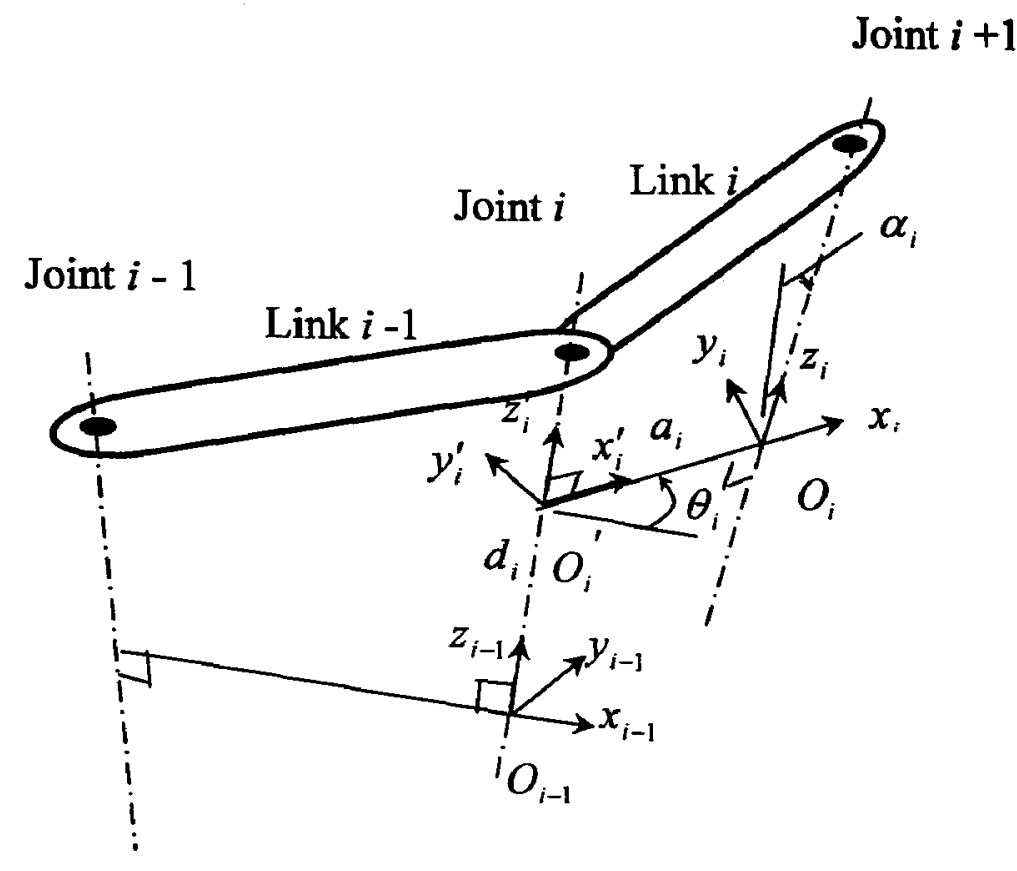

Figure 2.2 Generalized link coordinate system with Denavit-Hartenberg kinematic convention.

\subsection{Forward Kinematics Calculation}

Once the coordinate frames were assigned, from frame $\{0\}$ to frame $\{n\}$, the coordinate frame $\{e\}$ is also assigned at the end-point. Note that the base frame $\{b\}$ might not be necessarily the same as the frame $\{0\}$. Therefore, constant homogeneous matrices, $\boldsymbol{T}_{0}^{b}$ and $\boldsymbol{T}_{e}^{n}$, need to be calculated separately.

First, the homogeneous transformation matrix $\boldsymbol{T}_{n}^{0}$ can be calculated as follows

$$
\boldsymbol{T}_{n}^{0}(q)=\boldsymbol{T}_{1}^{0}\left(q_{1}\right) \boldsymbol{T}_{2}^{1}\left(q_{2}\right) \boldsymbol{T}_{3}^{2}\left(q_{3}\right) \ldots \boldsymbol{T}_{n}^{n-1}\left(q_{n}\right),
$$


to yield the pose of frame $\{n\}$ with respect to frame $\{0\}$. Then the forward kinematics will be calculated using:

$$
T_{e}^{b}(q)=T_{0}^{b} T_{I}^{o}\left(q_{1}\right) T_{2}^{1}\left(q_{2}\right) T_{3}^{2}\left(q_{3}\right) \ldots T_{n}^{n-I}\left(q_{n}\right) T_{e}^{n}
$$

to yield the pose of the end-effector frame with respect to the base frame.

\subsection{Workspace Calculation}

In robotics, the workspace is described by the origin of the end-point frame when all the robotic manipulator joints travel through all possible motions. One should differentiate the reachable and dextrous workspace. In the reachable workspace, the end-effector can exhibit at least one acceptable orientation, while with the dextrous workspace, the end-effector must be able to attain different orientations. Therefore the dextrous workspace is a subspace of the reachable workspace.

In general, the manipulator workspace is determined by the manipulator geometry and joint limits. Given the above definitions, the reachable workspace of a $n$ degrees-of-mobility manipulator is the geometric locus of the points that can be reached by forward kinematics, i.e.,

$$
\mathrm{W}=\boldsymbol{o}(q) \quad q_{i m} \leq q_{i} \leq q_{i M} \quad i=1,2, \ldots, n .
$$


Here, $q_{i m}$ and $q_{i M}$ are the minimum and maximum limits of joint $i$, respectively. The points $o(q)$ can be calculated from homogeneous transformation matrices. In the following chapter, the human arm will be modeled using robot kinematic notation and its workspace will be calculated accordingly. 


\section{Chapter 3}

\section{Kinematic Modeling of Human Arm}

In this chapter, a human arm will be modeled by adopting the $\mathrm{D}-\mathrm{H}$ convention used commonly for robot kinematic modeling. This is because the anatomy of the arm and joints are very complex and even if an exact anatomic model exists, it will not serve the objectives of this work. For kinematic modeling, kinematic pairs with a skeletal kinematic model of the arm would suffice. The rotational and translational motions of the joints can be modeled using revolute and prismatic joints, respectively. For example, a spherical joint of the shoulder could be modeled by three perpendicular revolute joints. In the following sections, a kinematic model of the human arm will be presented, and relevant $\mathrm{D}-\mathrm{H}$ parameters will be identified.

\subsection{Kinematic Structure}

The simplified kinematic structure of the arm is shown in Figure 3.1. The reach envelope can be approximated by considering only information about the shoulder and elbow joints. The shoulder joint has been modeled using three perpendicular revolute joints with joint variables 
$q_{1}, q_{2}, q_{3}$, resembling a spherical joint. Therefore, the motion of the glenohumeral joint has been limited to a spherical joint. The elbow joint has been also modeled using a revolute joint, with joint variable $q_{4}$. As noted in [16], there is no generalized biomechanical model of the shoulder, due to its anatomical complexity. Also, it is assumed that the joints motions are independent. In reality, the joint motions of the shoulder are dependent. The tip of the thumb will be marked as the point of interest. The tip of thumb has been assumed to be fixed with respect to the last coordinate frame.

Coordinate frames are assigned according to the $\mathrm{D}-\mathrm{H}$ convention. Here, $q_{1}$ is ExtensionFlexion of the shoulder, $q_{2}$ denotes the Abduction-Adduction (lateral-medial) of the shoulder, and $q_{3}$ represents Internal-External rotations of the shoulder. Finally, $q_{4}$ denotes the FlexionExtension of the elbow. 


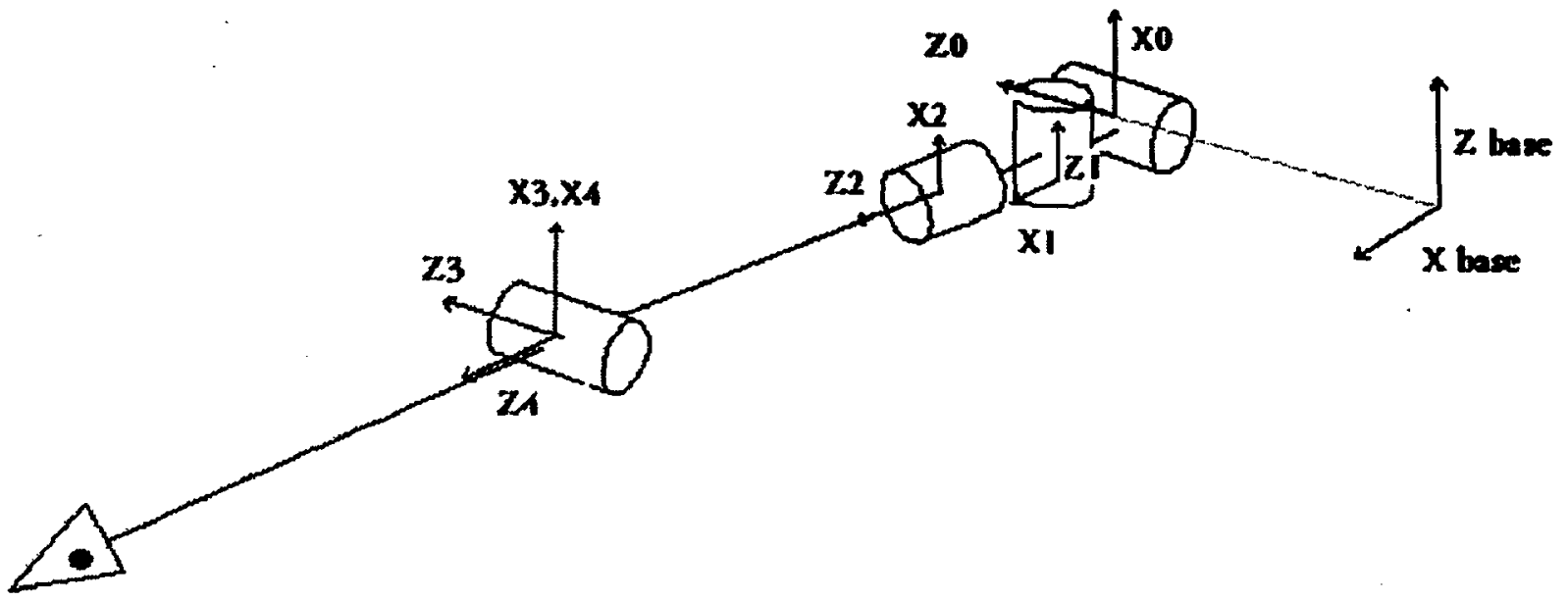

Point of Interest

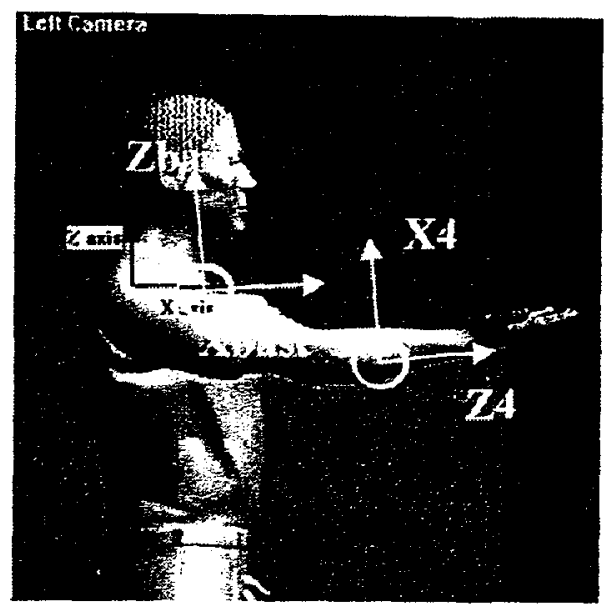

Figure 3.1 Kinematic modeling of human arm.

\subsection{Kineamtic Parameters}

The joint limits and D-H parameters were adopted using statistical biomedical data [29], [30], as follows.

$$
\begin{aligned}
& -151^{\circ}<q_{1}<98^{\circ}, \\
& -48^{\circ}<q_{2}<134^{\circ}, \\
& -34^{\circ}<q_{3}<97^{\circ},
\end{aligned}
$$




$$
-0^{\circ}<q_{4}<142^{\circ}
$$

and as shown in Table 3.1.

\begin{tabular}{|c|c|c|c|c|}
\hline $\begin{array}{c}\text { Joint } \\
\text { Number }\end{array}$ & $a$ & $\alpha$ & $d$ & $\theta$ \\
\hline 1 & 0 & $-\pi / 2$ & 0 & $q_{1}-\pi / 2$ \\
\hline 2 & 0 & $\pi / 2$ & 0 & $q_{2}+\pi / 2$ \\
\hline 3 & 0 & $-\pi / 2$ & $\begin{array}{c}\text { Upper Arm }(35 \\
\mathrm{cm})\end{array}$ & $q_{3}+\pi / 2$ \\
\hline 4 & 0 & $\pi / 2$ & 0 & $q_{4}$ \\
\hline
\end{tabular}

Table 3.1 The Kinematic parameters for 4-DOF arm model.

Finally, the point of interest (thumb tip) is located at $36 \mathrm{~cm}$ from the origin of coordinate

frame 4. The base coordinate frame is located at $-17.85 \mathrm{~cm}$ from the frame 0 (Figure 3.1).

\subsection{Forward Kinematics and Reach Envelope Algorithm}

Using the homogeneous transformation matrix given by equation (2.6), different frames can be related as follows.

$$
\boldsymbol{T}_{0}^{\text {base }}=\left[\begin{array}{cccc}
0 & -1 & 0 & 0 \\
0 & 0 & -1 & -17.85 \\
1 & 0 & 0 & 0 \\
0 & 0 & 0 & 1
\end{array}\right] \text {, }
$$




$$
\begin{aligned}
& \boldsymbol{T}_{1}^{\mathbf{0}}=\left[\begin{array}{cccc}
\sin \left(q_{1}\right) & 0 & \cos \left(q_{1}\right) & 0 \\
-\cos \left(q_{1}\right) & 0 & \sin \left(q_{1}\right) & 0 \\
0 & -1 & 0 & 0 \\
0 & 0 & 0 & 1
\end{array}\right], \\
& \boldsymbol{T}_{2}^{1}=\left[\begin{array}{cccc}
-\sin \left(q_{2}\right) & 0 & \cos \left(q_{2}\right) & 0 \\
\cos \left(q_{2}\right) & 0 & \sin \left(q_{2}\right) & 0 \\
0 & 1 & 0 & 0 \\
0 & 0 & 0 & 1
\end{array}\right], \\
& \boldsymbol{T}_{3}^{2}=\left[\begin{array}{cccc}
-\sin \left(q_{3}\right) & 0 & -\cos \left(q_{3}\right) & 0 \\
\cos \left(q_{3}\right) & 0 & -\sin \left(q_{3}\right) & 0 \\
0 & -1 & 0 & 35 \\
0 & 0 & 0 & 1
\end{array}\right], \\
& \boldsymbol{T}_{4}^{3}=\left[\begin{array}{cccc}
\cos \left(q_{4}\right) & 0 & \sin \left(q_{4}\right) & 0 \\
\sin \left(q_{4}\right) & 0 & -\cos \left(q_{4}\right) & 0 \\
0 & 1 & 0 & 0 \\
0 & 0 & 0 & 1
\end{array}\right] .
\end{aligned}
$$

According to equation (2.9), the problem of the reach envelope calculation will be:

$$
\mathrm{W}=\boldsymbol{o}(\boldsymbol{q}) \quad q_{i m} \leq q_{i} \leq q_{i M} \quad i=1,2, \ldots, n .
$$

The above equation can be simplified for the given model, as follows.

$$
\mathrm{W}=\left[\begin{array}{c}
x(q) \\
y(q) \\
z(q)
\end{array}\right]=\boldsymbol{T}_{4}^{\text {base }} p_{\text {thumb }}^{4} \quad \forall q_{i}, q_{i m} \leq q_{i} \leq q_{M M}
$$

where $p_{\text {thumb }}^{4}=[0,0,36]^{\mathrm{T}}(\mathrm{cm})$, and the joint limits are given by (3.1). Using equations (2.8), and (3.7), one can calculate: 


$$
\begin{aligned}
& x=36\left(\cos \left(q_{1}\right) \sin \left(q_{1}\right) \sin \left(q_{3}\right)-\sin \left(q_{1}\right) \cos \left(q_{3}\right)\right) \sin \left(q_{4}\right)+36 \cos \left(q_{1}\right) \cos \left(q_{2}\right) \cos \left(q_{4}\right)+35 \cos \left(q_{1}\right) \cos \left(q_{2}\right), \\
& y=-17.8-36 \cos \left(q_{2}\right) \sin \left(q_{3}\right) \sin \left(q_{4}\right)+36 \sin \left(q_{2}\right) \cos \left(q_{4}\right)+35 \sin \left(q_{2}\right), \\
& z=36\left(\sin \left(q_{1}\right) \sin \left(q_{2}\right) \sin \left(q_{3}\right)+\cos \left(q_{1}\right) \cos \left(q_{3}\right)\right) \sin \left(q_{4}\right)+36 \sin \left(q_{1}\right) \cos \left(q_{2}\right) \cos \left(q_{4}\right)+35 \sin \left(q_{1}\right) \cos \left(q_{2}\right) .
\end{aligned}
$$

The above set of equations (3.8) determines the reach envelope for the thumb tip. That is the set of all points touchable by the tip of the thumb.

In summary, the algorithm for the human arm reach envelope calculation will follow the algorithm shown in Figure 3.2. 


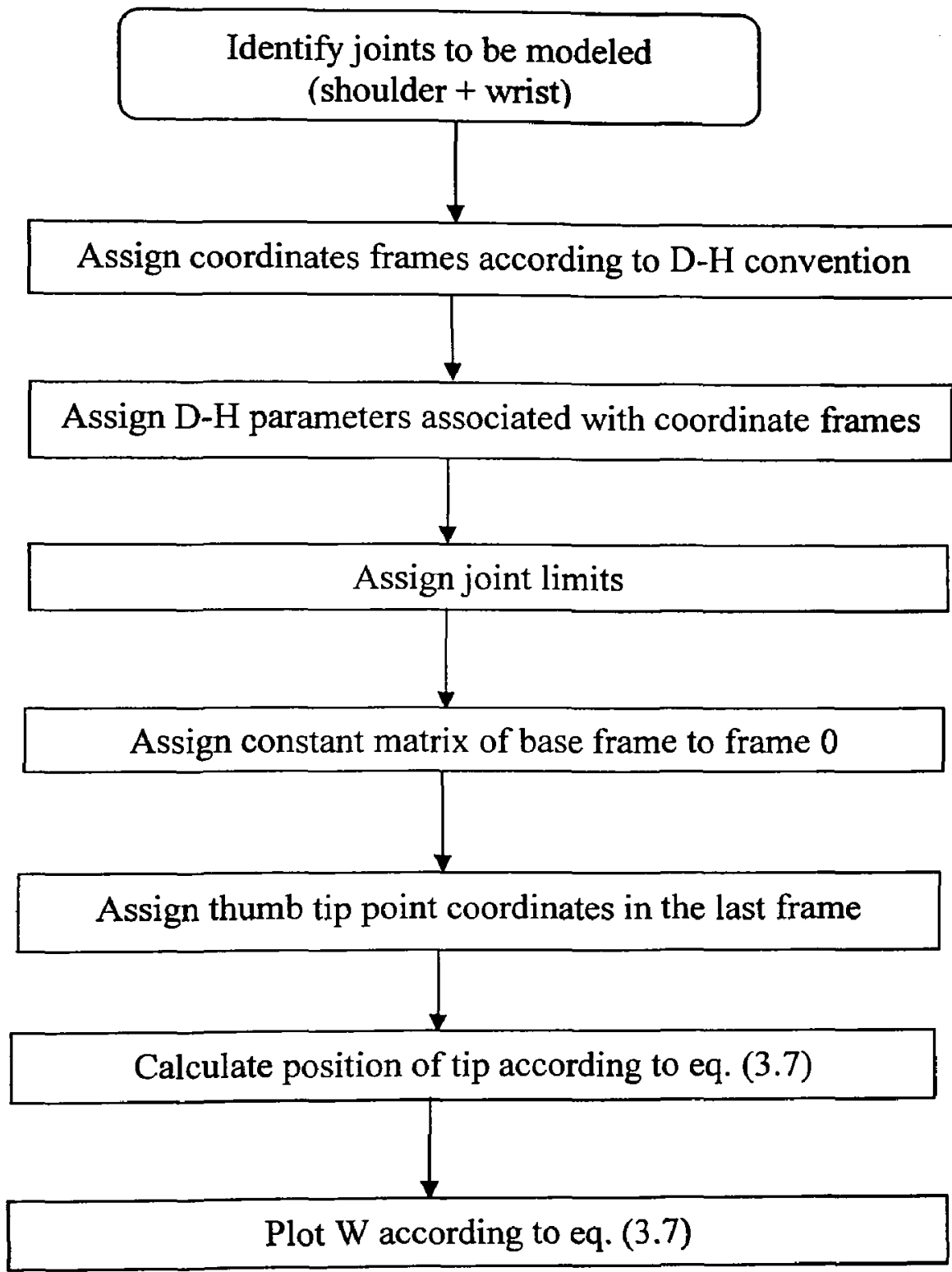

Figure 3.2 Human arm envelope calculation algorithm 


\subsection{Simulation Results}

Simulations were run to show the human arm reach envelope. The parameters were chosen from [29], [30]. Programs were written in Maple ${ }^{\mathrm{TM}} 3.1$ and MatlabTM 6.5 and run under Windows XP using a P4-1.7 GHz PC to simulate the modeled arm reach. For computational efficiency, a resolution (computation step) of $0.5 \mathrm{~cm}$ was considered. This tolerance is compatible with similar studies in previous work [8]. The results are shown in Figures 3.3-3.7. For ease of visualization, envelope boundaries are shown in horizontal $\mathrm{XY}$ and vertical $\mathrm{Z}$ planes.

The results are only shown for the right arm. However, the results could be extended to both arms. The solid model of the human body has not been considered for possible collision and intersection. This can be readily done using a solid modeler and incorporating the boundaries at different $y$ and $z$ planes. The following observations could be made.

First, the normal horizontal working area (Figures 3.3-3.4) can be well-predicted by the proposed method. It is seen that as the depth from the shoulder increases (in the negative $z$ direction), the horizontal working area improves. The study has been limit to the investigation of the practical and low depth working tables (i.e., $-45 \leq z \leq 0 \mathrm{~cm}$ ). The results are consistent with the previous experimental results of [20], [18], [19]. As it is seen, after $z=-27 \mathrm{~cm}$, there is 
no significant change in the reach envelope, except that envelope shifts in a minor way towards the other arm.

Second, in contrast to many of the previous experimental methods, e.g., [18], the results also show the rear reach area. This is, of course, not usually practically important. However, in certain operations, it might be required to investigate the rear reach as well. The method allows prediction of such working areas. As seen from Figures (3.3-3.4), the rear reach improves with negative distance from the shoulders.

Third, the changes in the vertical working areas ( $y$ planes) are not as significant when compared to changes in the horizontal working areas (Figures 3.6-3.7). It is worth noting that many of the previous methods have ignored the study of the vertical working spaces. The proposed method can easily provide the reach envelope at any vertical plane. 


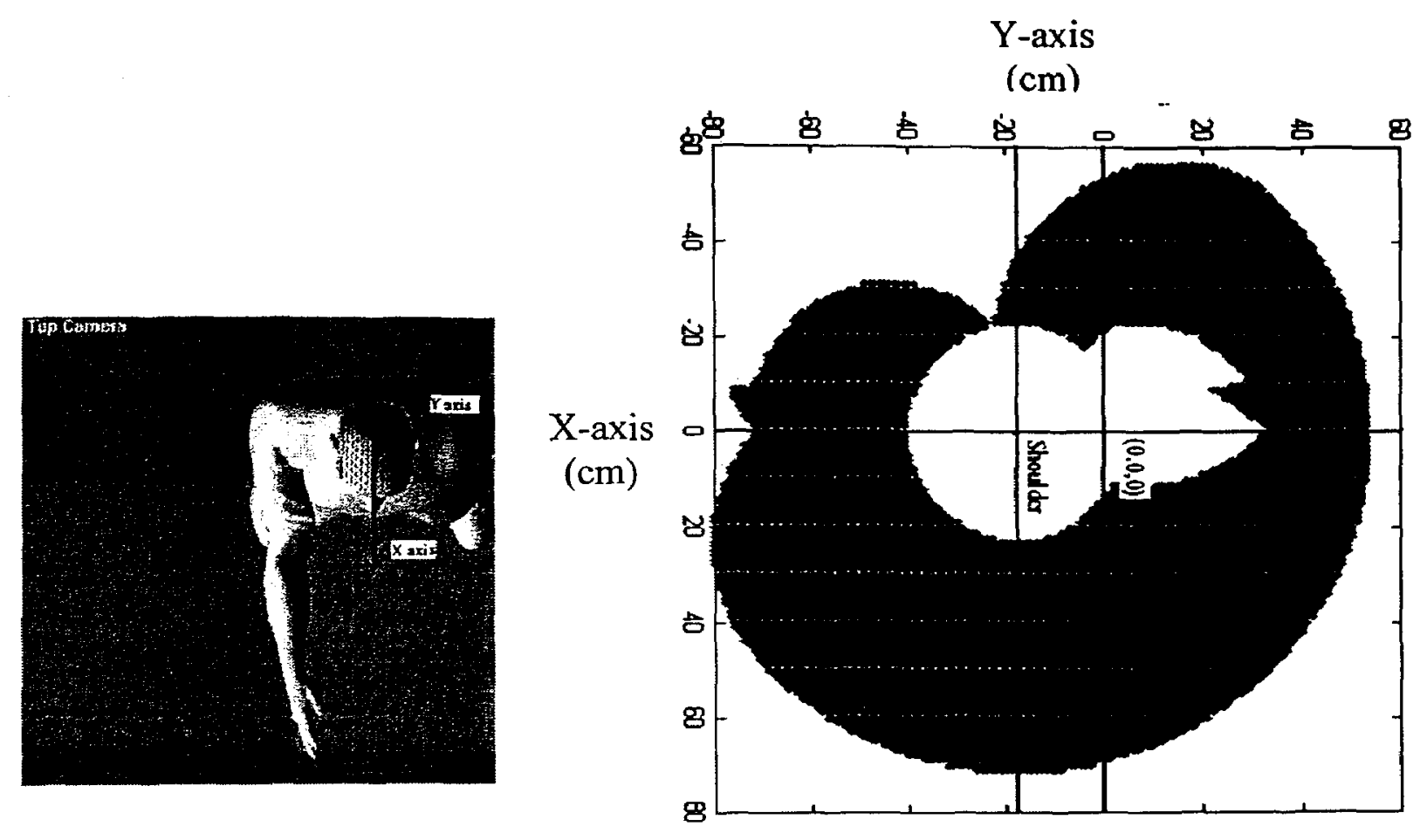

Figure 3.3 Reach envelope at $z=0$ plane of the base frame (all units in $\mathrm{cm}$ ). 


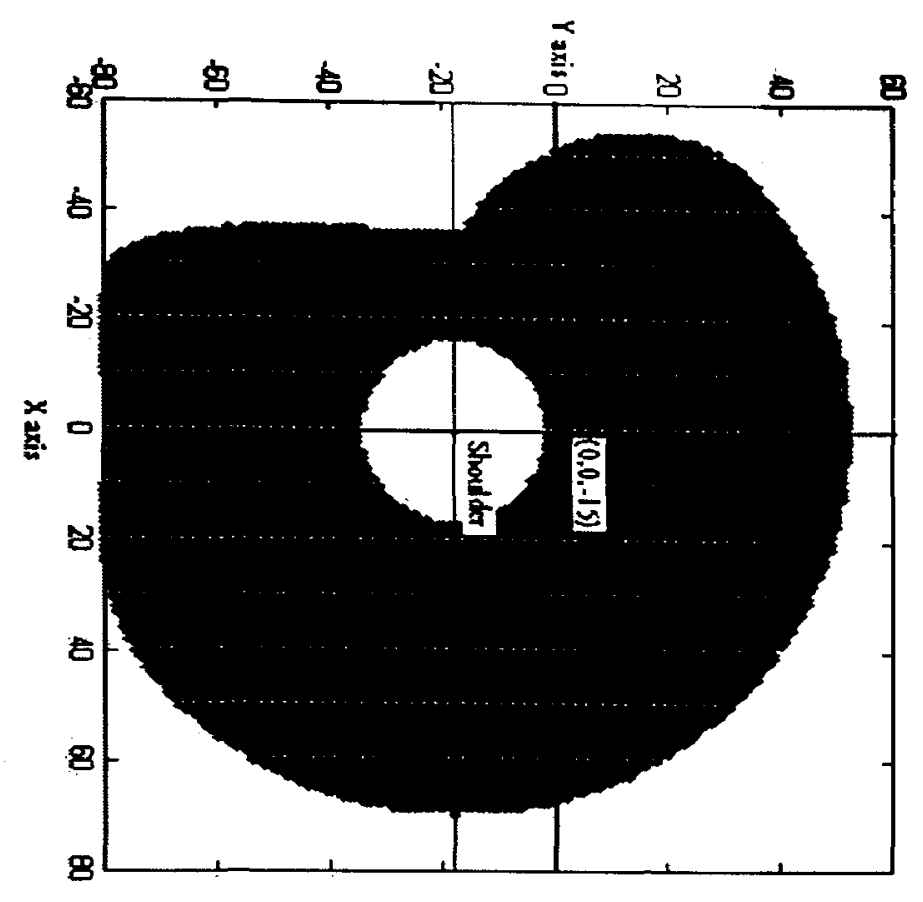

(a)

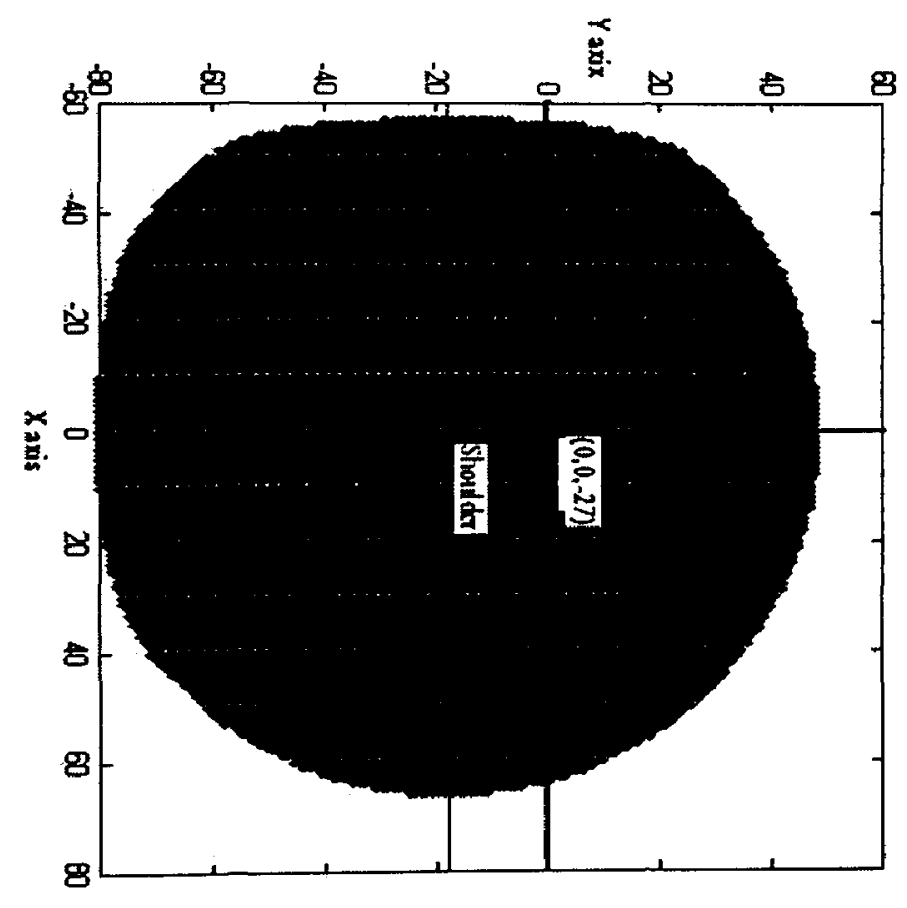

(b)

Figure 3.4 Reach envelope at (a) $z=-15$, and (b) $z=-27$ of the base frame (all units in $\mathrm{cm}$ ). 


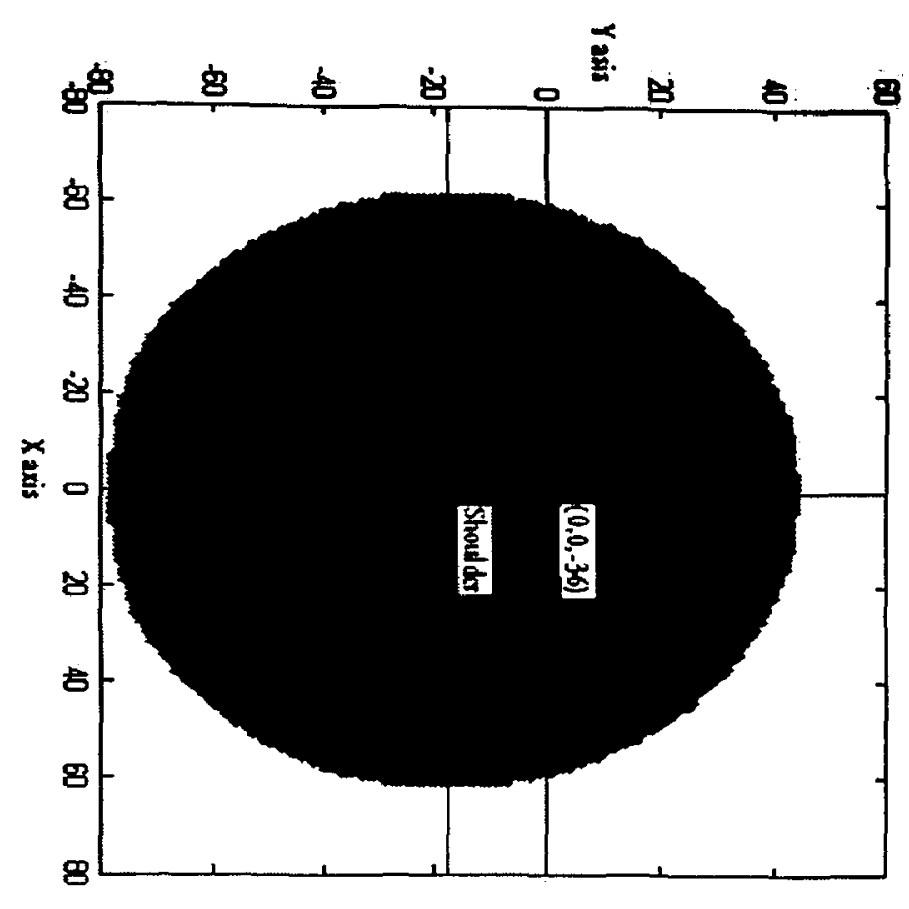

(a)

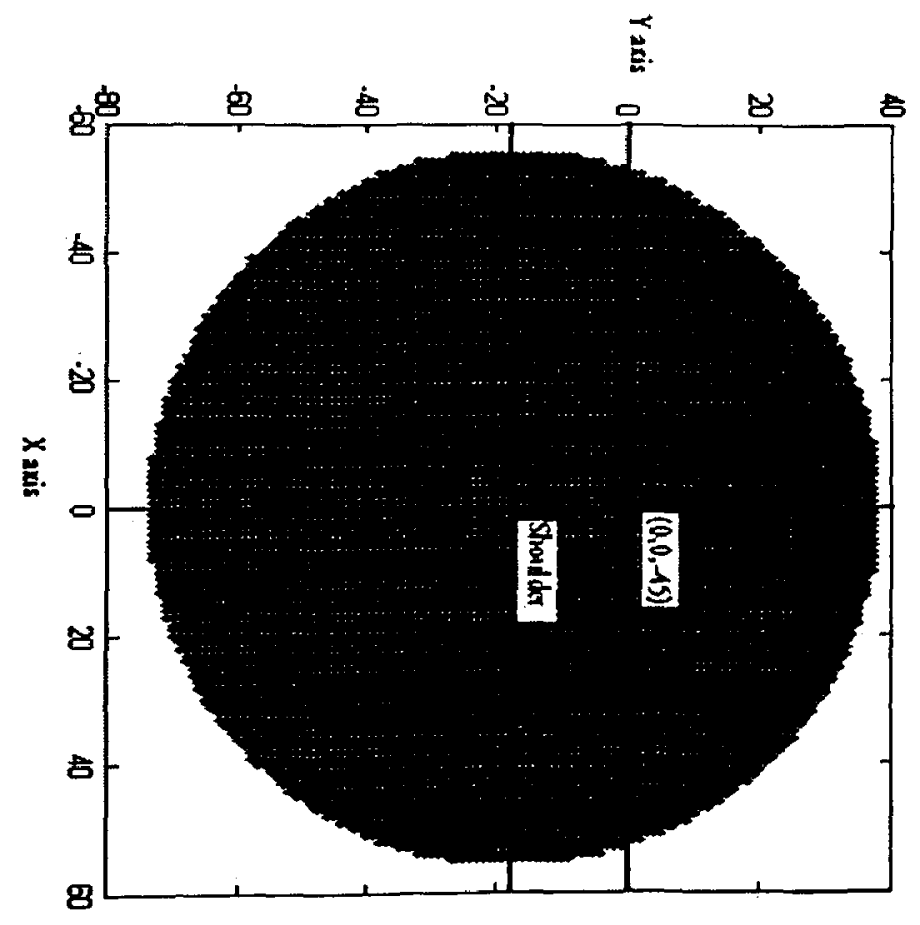

(b)

Figure 3.5 Reach envelope at (a) $z=-36$, and (b) $z=-45$ of the base frame (all units in cm). 

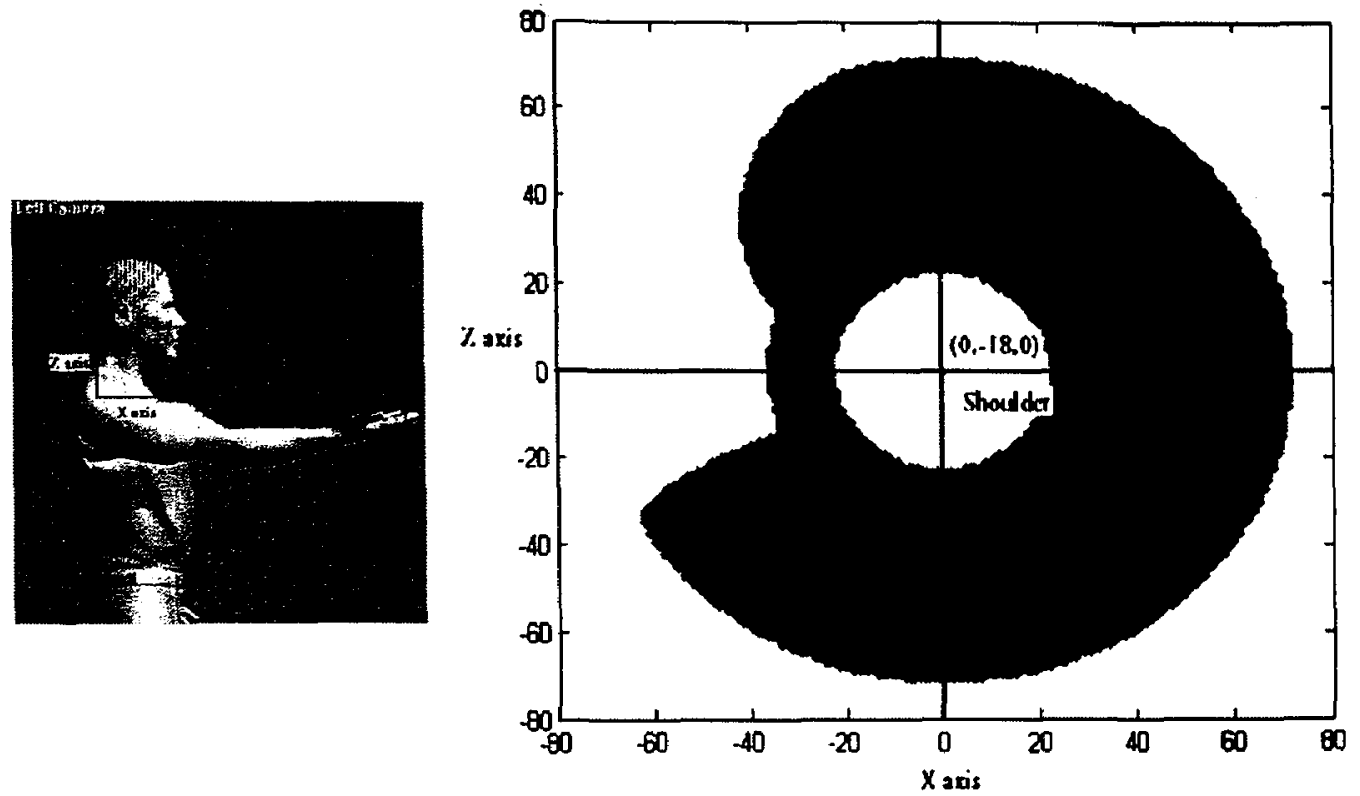

Figure 3.6 Reach envelope at $y=0$ plane of the base frame (all units in $\mathrm{cm}$ ). 


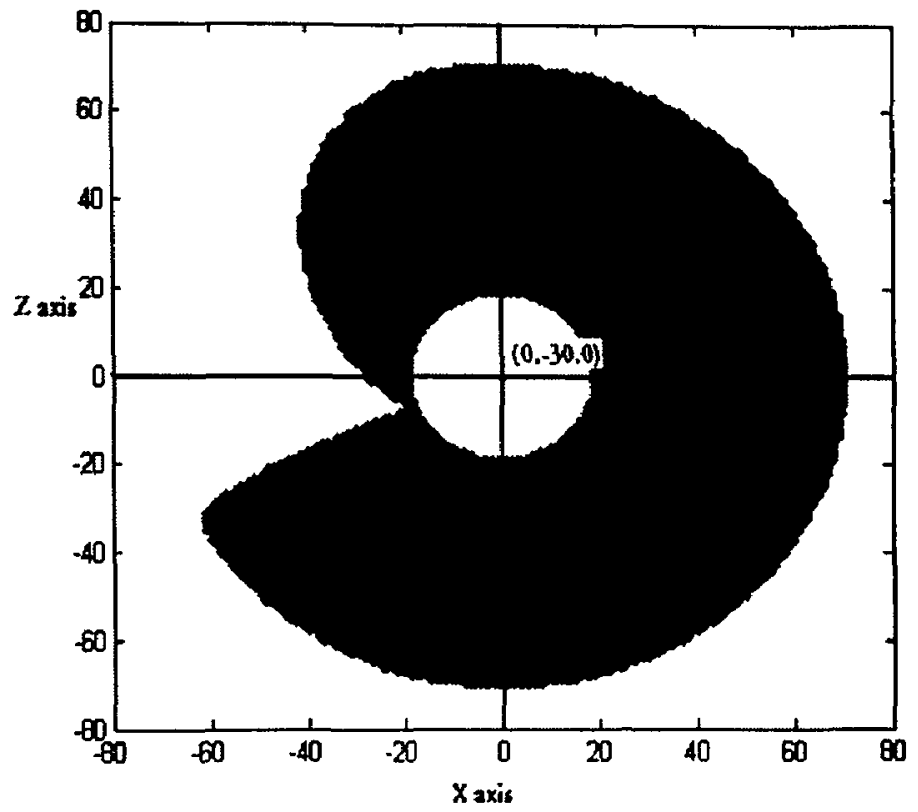

Figure 3.7 Reach envelope at $y=-30$ plane of the base frame (all units in $\mathrm{cm}$ ). 


\section{Chapter 4}

\section{Conclusions and Future Work}

Calculation and visualization of the human arm envelope could serve many practical workspace designs such as cockpit and automobile interior designs. It could also help physiotherapists, occupational therapists, neurologists, and the medical community to understand neural strategies to allow posing hand during voluntary reaching movements and to quantifying the functional potential of a joint. This in turn, will facilitate the selection of ergonomic postures to reduce joint stresses.

In this work, a human arm was modeled using a D-H convention adopted from robot kinematics. The $\mathrm{D}-\mathrm{H}$ technique is based on four parameters update and four transformation matrices. Therefore, the minimal representation makes the calculation and computation simpler and more efficient. The developed model was then used to calculate the arm reach envelope in 3D. For this purpose, statistical real data of the female firefighters in UK [29] was utilized. However, the methodology can easily allow incorporation of any individual arm data. The individual joint limits were also integrated. In particular, the presented framework meets the main characteristics dișcussed in the Chapter 1, including the following: 
- Could be readily adapted to many tasks and workspace designs.

- Integrates individual data rather than average population data.

- Considers major degrees of freedom of the arm.

- Does not require expensive tools or computing systems, and could run on regular PCs.

- Allows the user to enter required data and determine the workspace readily.

The simulation results indicated effectiveness and accuracy of the developed method. Some observations were also made with respect to the reach envelope changes in depth ( $z$-direction) and width (y-direction).

Some issues, however, remain to be addressed. One is self-collision detection. The present system cannot detect collision and interference of the human arm with other parts of body. Those poses that cause self-collision must be discarded. two is to extend the degrees of freedom of the present system to present the human arm more realistically. It seems that 9 degrees-offreedom is required to reflect all motions of the arm from shoulder base to thumb. Three includes the extension of the present (positioning) work to the study of the orientation workspace. In particular, calculation of dextrous workspace is important for many workspace design applications. Four is the integration of joint fatigue in the calculation of reachable workspace. Studies have shown that joints fatigue would affect the joint limits. Five is the joints limits dependence that has been ignored in this study. Future work of this group must address the above shortcomings. 


\section{References}

[1] D.B. Chaffin, G.B.J. Andersson, B.J. Martin, Occupational Biomechanics, New York, Wiley, 3rd edition, 1999.

[2] A. Grieco, "Application of the concise exposure index (OCRA) to tasks involving repetitive movements of the upper limbs in a variety of manufacturing industries: preliminary validation," J. Egonomics, vol. 41, no. 9, pp. 1347-1356, 1998.

[3] Y.S. Kim, L.R. Yeh, D. Trudell, and D. Resnick, "MR Imaging of the major nerves about the elbow," Skeletal Radiology, vol. 27, pp. 419-426, 1998.

[4] C.M. Burns, K.J. Vicente, K. Christoffersen, W.S., Pawlak, "Towards viable, useful, and usable human factors design guidance," Elsevier J. Applied Ergonomics, vol. 28, no. 5, pp. 311 $322,1997$.

[5] R. Feyan, Y. Liu, D. Chaffin, G. Jimmerson, and B. Joseph, "Computer-aided ergonomics: a case study of incorporating ergonomic analyses into workspace design," Elsevier J. Applied Ergonomics, vol. 31, pp. 291-300, 2000.

[6] X. Wang, J.-P. Verriest, "A geometric algorithm to predict the arm reach posture for computer-aided ergonomic evaluation", J. of Visualization and Computer Animation, vol. 9, pp. $33-47,1998$.

[7] J.M. Porter, M. Freer, K. Case, and M.C. Bonney, "Computer-aided ergonomics and workspace design," in Evalauation of Human Work: A Practical Ergonomics Methodology, J.R. Wilson, and E.N. Colett, (Eds.), $2^{\text {nd }}$ Edition, Taylor and Francis, London, pp. 574-620, 1995. 
[8] E.S. Jung, D. Kee, and M.K. Chung, "Upper body reach posture prediction for ergonomic evaluation models," Int. J. Industrial Ergonomics, vol. 16, pp. 95-107, 1995.

[9] J. Denavit and R.S. Hertenberg, "A kinamtic notation for lower pair mechanisms," J. Applied Mechanics, vol.22, pp. 215-221, 1955.

[10] R.R. Farley, "Some principles of methods and motion study as used in development work," General Motors Engineering Journal, vol. 2, pp. 20-25, 1955.

[11] C.H. Yu, and N.N. Donaldson, "Measurement of posture by triangulation using potentiometers," J. Med. Eng. Phys., vol. 21, no. 4, pp. 259-263, 1999.

[12] D.J. Reinkensmeyer, J.P. Dewald, W.Z., Rymer, "Guidance-based quantification of arm impairment following brain injury: a pilot study," IEEE Trans. Rehabil. Eng., vol. 7, no. 1, pp. 1$11,1999$.

[13] AMA 1997, Guides to the Evaluation of Permanent Impairment, American Medical Association, Chicago, IL, 1997.

[14] J. Richards, "The measurement of human motion: A comparison of commercially available systems," Proc. $5^{\text {th }}$ Int. Symp. 3-D Analysis of Human Movement, Chabanacoque, Tennessee, 1998.

[15] J.J. Schillings, R.G. Meulenbroek, and A.J. Thomassen, "Functional properties of graphic workspaces: assessment by means of a 3D geometric arm model," Acta Psychol (Amst), vol. 100, no. 1-2, pp.97-115, 1998 .

[16] K. Abdel-Malek, J. Yang, M. Vennier, R. Brand, and E. Tanbour, "Towards understanding the workspace of human limbs," Int. J. Ergonomics, pp. 63-72, 2002. 
[17] R.W. Faulkner, and R.A. Day, "The maximum functional reach for the female operator," AIIE Trans., vol. 2, pp. 126-131, 1970.

[18] B. Das, and D.N. Behara, "Three-dimensional workspace for industrial workstations," $J$. Human Factors, vol. 40, no. 4, pp. 633-646, 1998.

[19] P. Squires, "The shape of the normal work area," Report no. 275, Navy Dept., Medical Research Lab., New London, Connecticut, USA.

[20] B. Das, and D.N. Behara, "Determination of the normal horizontal working area: a new model and method," J. Ergonomics, vol. 38, no. 4, pp. 734-748, 1995.

[21] N. Nayar, "Deneb/ERGO-a simulation based human factors tool," Winter Simulation Conf. Proc., pp. 427-431, 1995.

[22] L.W. O'Sullivan, and T.J. Gallwey, "Relationship of upper limb postures to anthropometric variables," in M.A. Hanson, E.J. Lovesey, and S.A. Robertson (Eds.), Contemporary Ergonomics 2000, Proc. Ergonomic Soc. Annual Conf., Taylor and Francis, London, pp. 44-48, 2000

[23] N.J. Badler, C.B. Phillips, B.L. Webber, Simulating Humans: Computer Graphics, Animation, and Control, Oxford University Press, 1993.

[24] D.H. Kee, Y.T. Shin, D.S. Kang, and E.S. Chung, "Reach volume," Proc. Fall Conf. Korean Inst. Industrial Engineers, pp. 232-237, 1996.

[25] K. Abdel-Malek, and H.J. Yeh, "Analytic boundary of the workspace for general three degree of freedom mechanisms," Int. J. Robotics Research, vol. 16, no. 2, pp. 198-213, 1997.

[26] M.W. Spong and M. Vidyasagar, Robot Dynamics and Control, Wiley, New York, 1989.

[27] H. Asad and J.E. Slotine, Robot Analysis and Control, John Wiley, New York, 1986. 
[28] J.J. Craig, Introduction to Robotics, Addison Wesley, Reading, MA, 1989.

[29] M. Stirling, National Anthropometry Survey of Female Firefighters, UK: the Chief Fire Officers Association, CACFOA, July 2002.

[30] S. Kousidou, N.G. Tsagarakis, and D. G. Caldwell, "Evaluation of a soft exoskeleton for rehabilitation and physiotherapy of the upper limb," Proceedings of ICAR 2003,The 11th International Conference on Advanced Robotics, pp. 1080-1085, 2003.

[31] L.W. O'Sullivan, and T.J. Gallwey, "Effects of gender and reach distance on risks of musculoskeletal injuries in an assembly task," Elesvier Int. J. Industrial Ergonomics, vol. 29, pp. $61-71,2002$. 
